\title{
Cellular Haemostaseology - Diagnosis and Therapeutic Monitoring of Inherited and Acquired Functional Platelet Disorders
}

\author{
Mariam Klouche \\ Bremer Zentrum für Laboratoriumsmedizin und LADR GmbH Medizinisches Versorgungszentrum Bremen, Germany
}

Platelets execute a key function in the regulation of haemostasis, clot stability and retraction, vascular tone and repair as well as in host defence. In exerting their function, platelets, as the central elements of cellular haemostasis, show a finetuned, intricate, activation state-dependent interaction with mediators of the plasmatic coagulation system and platelet activators, with circulating and resident cells such as leucocytes, platelets themselves, endothelial and vascular smooth muscle cells as well as with the subcellular matrix. Platelet activation is a tightly regulated process, allowing the immediate recognition of vascular damage leading to platelet activation and ultimately to formation of the primary haemostatic plug, a process which normally is self-limited and under straight control. Consequently, minor disturbances of this balanced homeostasis may result in pronounced platelet dysfunction, which has a major impact on many pathophysiological processes such as thrombosis, haemorrhage, inflammation, atherogenesis and cardiovascular disease but also on tumour growth and metastasis as well as on the anti-microbial host defence. Alterations of the functional capacity of platelets have been associated with the prognosis and severity of distinct clinical diseases, including cardiovascular diseases, diabetes, tumour metastasis and sepsis. Even though primary (hereditary) platelet functional abnormalities are very rare disorders, acquired platelet function abnormalities are relatively frequent and occur in the context of many diseases and may be mediated iatrogenically by therapeutic administration of drugs. The clinical presentation of primary or secondary platelet function disorders is very variable. Given the importance of platelets for cellular haemostasis, the recognition of platelet function disturbances, the impact of therapeutic suppression during treatment with anti-platelet medications as well as the proper selection of diagnostic tests for diagnosis and for their application as preventive risk markers is of utmost relevance for rational clinical use.
In this first part of reviews on 'cellular haemostaseology, Kerstin Jurk and Beate Kehrel [1] provide an eminent exhaustive classification of inherited and acquired disorders of platelet function. Not only are the clinical features described in detail, but also the pathophysiology and the involved defect are clearly outlined, enabling the development of a systematic diagnostic schedule. A comprehensive overview on the methodological principles of platelet function analysis encompassing the selective analysis of distinct aspects of platelet function and global function tests in conjunction with the currently available diagnostic devices is presented in the review of Mariam Klouche [2]. In addition, the application and suitability of the different test systems for the diagnosis of distinct platelet function disorders, including the restrictions of each method, is presented to promote an optimal selection of diagnostic tests. Wolfgang Lösche and Stan Heptinstall [3] give an excellent overview on the value of platelet activation markers as prothrombotic and thromboembolic risk indicators, and provide a thorough presentation of the currently available information from clinical and preclinical studies, which allows a knowledge-based estimation of the clinical usability of each predictive parameter. Particular focus is given to the diagnostic value of the different approaches to measure platelet reactivity and platelet activation and to pre- and peri-analytical considerations to ascertain the quality. Ingo Ahrens, Meike Schwarz, Karlheinz Peter and Christoph Bode [4] give a comprehensive overview on the current pharmacological strategies for the inhibition of platelet function. The review encompasses in depth information on the mechanisms of action and the clinical use of classic approved platelet inhibitors, up to the most recently released new classes of anti-platelet agents, such as platelet integrin blockers including substances under development. The principle of action is described in detail, allowing a sound estimation of the currently available and evolving methods to diagnose platelet function disorders. Fi-

\begin{tabular}{ll}
\hline KARGER & ( ) 2007 S. Karger GmbH, Freiburg \\
Fax +49 7614520714 & Accessible online at: \\
$\begin{array}{l}\text { E-mail Information@Karger.de } \\
\text { www.karger.com }\end{array}$ & www.karger.com/tmh \\
&
\end{tabular}

Prof. Dr. med. Mariam Klouche 
nally, Werner Streif together with Martin Olivieri, Stefan Weickardt, Wolfgang Eberl, Ralf Knoefler [5] give a very informative review on the results of the Thromkid competence network for functional platelet abnormalities of the Paediatric Group of the German Thrombosis and Haemostasis Research Society (GTH). The survey is based on the German-speaking population and allows for the first time a thorough insight in the commonly used methods and diagnostic strategies applied for the detection of functional platelet disorders. Together this series of reviews provides the state of the art knowledge on the epidemiology of hereditary and acquired functional platelet disorders, on the diagnostic principles, including a rational diagnostic algorithm with an additional outline of the most frequently applied methods in German-speaking countries, on the predictive value of distinct platelet function tests for thromboembolic disorders and on the impact of the currently applied therapeutic inhibitors of platelet function.

\section{References}

1 Jurk K, Kehrel BE: Inherited and acquired disorders of platelet function. Transfus Med Hemother 2007;DOI 10.1159/000098178

2 Klouche M: Diagnostic Methods for Platelet Function Analysis. Transfusion Med Hemother 2007 DOI: 10.1159/000098179

3 Lösche W, Heptinstall S: Value of platelet activation markers as prothrombotic risk indicators. Transfus Med Hemother 2007;DOI: 10.1159/000097497

4 Ahrens I, Schwarz M, Peter K, Bode C: Therapeutic inhibitors of platelet aggregation - from aspirin to integrin blockers. Transfusion Med Hemother 2007;DOI $10.1159 / 000097664$

5 Streif W, Olivieri M, Weickardt St, Eberl W, Knoefler R, for the members of the Thromkid study group of the GTH: Thromkid - a competence network for functional platelet abnormalities. Transfusion Med Hemother 2007;DOI 10.1159/ 000097848 . 